\title{
México: entorno macroeconómico y mercados laborales
}

XLV Reunión Trimestral del Centro de Modelística y Pronósticos Económicos

\section{Ricardo Arriaga Campos*}

El pasado 26 de agosto de 2014, en el Auditorio Narciso Bassols de la Facultad de Economía y con una transmisión en vivo (a través de la liga http://www. economia.unam.mx/) el Centro de Modelística y Pronósticos Económicos (Cempe) llevó a cabo la Xuv Reunión Trimestral (Tercera Reunión 2014), con la temática "México: entorno macroeconómico y mercados laborales". En esta edición, moderada por Hugo Contreras Sosa, participaron los ponentes: José Gabriel Cuadra García, investigador económico de la Dirección General de Investigación Económica del Banco de México, quien ofreció la ponencia "La economía mexicana ante la normalización de la política monetaria en Estados Unidos"; César Castro, representante del grupo DARSI (consultora en investigación de mercados), que expuso el "Impacto macroeconómico de las reformas estructurales", así como el propio coordinador del Cempe, Eduardo Loría, que desglosó el tema "México: mercados laborales", así como los pronósticos para la economía mexicana.

\section{Implicaciones del retiro del estímulo monetario en economías avanzadas}

Cuadra García anticipó la relevancia del tema de los estímulos monetarios para las economías emergentes, in- cluido México: el retiro del estímulo monetario en economías avanzadas, particularmente en Estados Unidos, y cuáles son las implicaciones para economías pequeñas, abiertas, emergentes como la mexicana. Como antecedente, recordó que al inicio de la crisis financiera internacional de 2007 y a partir de su intensificación en 2008 con el colapso del banco de inversión Lehman Brothers, las economías avanzadas se han distinguido por tener brechas del producto negativas, por tener una posición fiscal frágil y por la necesidad de apoyar la actividad económica en estos países, de modo que las condiciones monetarias se han mantenido en una forma excepcionalmente acomodaticia; los bancos centrales de estos países respondieron rápidamente reduciendo las tasas de política a niveles cercanos a cero, además de a través de la implementación de las llamadas políticas monetarias no convencionales, las cuales corresponden en grandes rasgos a los programas de compras de activos que tuvieron la característica de modificar la composición y tamaño de las hojas de balance. Adicionalmente se aplicaron estrategias de comunicación proporcionando información más explícita sobre la trayectoria futura de las tasas de política con el propósito de influir en las tasas de interés de mediano y largo plazos.

\footnotetext{
* Profesor de la Facultad de Economía, UNAM . - .
} 
El investigador del Banco de México acotó que tales acciones de política monetaria tuvieron como finalidad en un primer momento restablecer el funcionamiento ordenado de los mercados financieros en estos países y posteriormente proporcionar un estímulo monetario adicional una vez que las tasas de política monetaria se encontraban en niveles cercanos a cero. En términos generales se puede decir que dichos estímulos fueron sustanciales y se puede afirmar que esas políticas contribuyeron a impedir que la recesión se transformara en una gran depresión.

\section{Las economías avanzadas se encuentran en fases distintas del ciclo económico}

Por otro lado, señaló que la evolución del producto interno bruto y la dinámica del mercado laboral de las economías avanzadas han mostrado un comportamiento mixto, por ejemplo en Estados Unidos la recuperación ha sido mayor que en otras regiones como la Zona Euro, y básicamente, a pesar de que la recuperación no se ha consolidado, es aún frágil, el desempeño de la economía estadounidense ha pasado por una contracción económica a principios de año sobre todo por factores climáticos, pero después se ha dado un retorno a una trayectoria positiva y a una disminución en la tasa de desempleo. El mensaje que sintetizó en las gráficas expuestas fue que las economías avanzadas se encuentran en fases distintas del ciclo económico y distintas posturas de política monetaria.

\section{Importante impacto en las economías emergentes}

Como consecuencia de las dinámicas descritas en las economías avanzadas, particularmente en Estados Unidos continuó Gabriel Cuadra-, ha habido un impacto importante en las emergentes: se ha generado un entorno de amplia liquidez en la economía global que contribuye a un periodo de importantes flujos de capital hacia las segundas. Los motivos de estos flujos corresponden en parte a las tasas de interés excepcionalmente bajas en las avanzadas y con ello un proceso de búsqueda de rendimientos por parte de inversionistas globales, un mayor apetito por riesgo. Otro factor es el hecho de que en ese momento las perspectivas económicas eran más favorables en las emergentes. En las economías receptoras, aquellos flujos de capital generaron preocupación por la apreciación excesiva del tipo de cambio nominal y una apreciación real que impacta las exportaciones netas. Otra preocupación fue el aumento y formación de burbujas en los precios de los activos, así como expansiones excesivas, insostenibles, del crédito hacia el sector privado, por 
lo cual esos flujos de capital pueden estar asociados con una mayor vulnerabilidad de los sistemas financieros domésticos que puede dar lugar a problemas de desalineación de los precios de los activos o episodios de expansión desmedida del crédito. Asimismo, dijo, se puede hablar de que si bien esas eran las preocupaciones en una primera etapa, también es cierto que a partir del año pasado, de mayo de 2013 cuando se empieza a plantear la posibilidad de que se empiecen a retirar los estímulos monetarios en Estados Unidos, como anuncio sorpresivo, se generó un periodo de volatilidad en los mercados financieros internacionales y en ese momento aumentó la preocupación sobre una reversión abrupta de los flujos de capital, lo que provoca como consecuencia una contracción en la absorción doméstica en la actividad económica, una depreciación importante del tipo de cambio real y una contracción en el crédito hacia el sector privado, una reversión repentina en las cuentas externas, en particular en la cuenta corriente, y con ello la posibilidad de un impacto sobre la estabilidad financiera de las economías receptoras y -advirtió- no se puede descartar un episodio de volatilidad elevada.

\section{En el contexto descrito, es recomendable seguir un enfoque integral de política.}

En este entorno de volatilidad y bajo crecimiento a nivel global

México ha seguido un enfoque basado en 3 pilares:

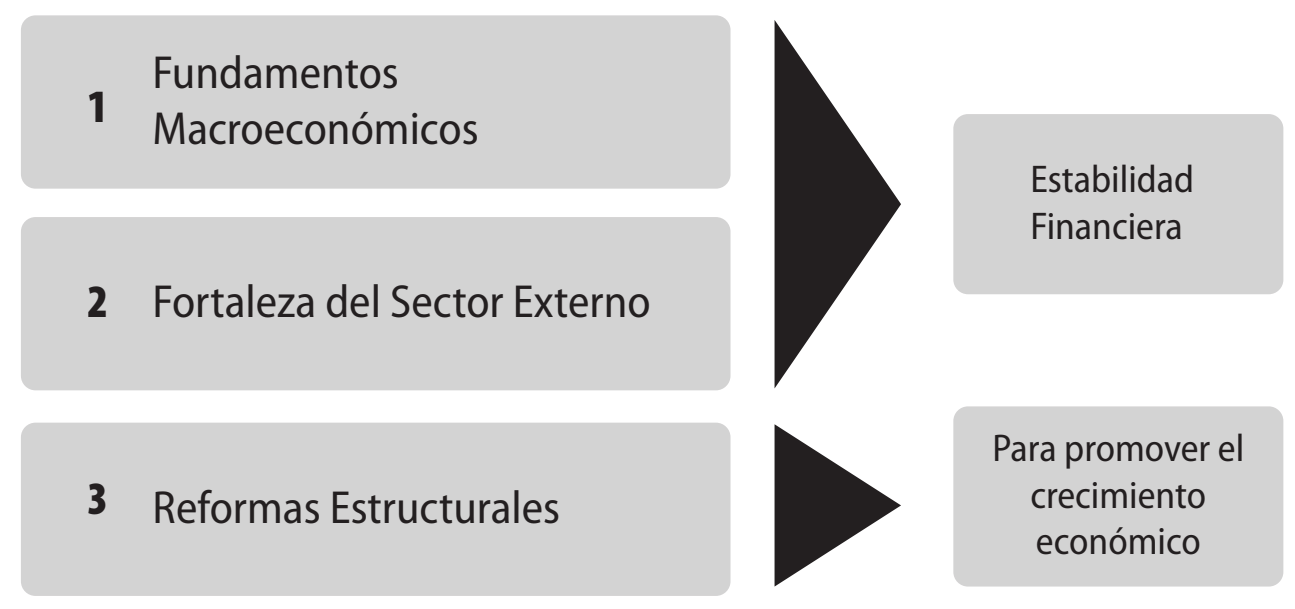


Economía Informa núm. 388 septiembre - octubre • 2014 | " " " " " " " " - " "

\section{Hoy, la pregunta central es cómo deben prepararse las economías emergentes}

A partir del segundo trimestre de este año se ha dado un nuevo ímpetu de capitales hacia las economías emergentes. En este entorno, hoy la pregunta central para las autoridades de estas economías, es cómo prepararse o cómo actuar ante la posibilidad de que este retiro se lleve a cabo no de manera gradual, ordenada, sino que pudiera darse una etapa de alta volatilidad. La gran pregunta es cómo estar listos para enfrentar un escenario internacional complejo, difícil. Para nosotros hay distintos pilares que pueden ayudar a reducir la volatilidad externa, como los fundamentos macroeconómicos, la fortaleza del sector externo y la adopción de reformas encaminadas a promover el crecimiento económico tomando en consideración la importancia de fortalecer las fuentes internas de crecimiento.

\section{Los objetivos oficiales: muy optimistas}

El siguiente expositor de la reunión del Cempe, el actuario César Castro, representante de DARSI (www. darsi.com.mx), grupo especializado en inteligencia de mercados, partió de la exposición de los propósitos del gobierno mexicano para aumentar la productividad y la competitividad, atraer inversión y alcanzar un crecimiento del cinco por ciento. Así, su ponencia giró en torno a las preguntas de si es posible alcanzar, con las reformas estructurales, un crecimiento sostenido de mediano y largo plazo de $5 \%$ anual.

En México, dijo el también creador -junto con Eduardo Loría, coordinador del Cempe- del modelo Eudoxio para las proyecciones económicas trimestrales, se considera que la economía necesita generar al menos un millón de empleos, esto en apego a una regla que establece que por cada uno por ciento de crecimiento se supone que se deben generar 200 mil empleos, aunque -advirtió- habría que ver en el mundo cuántos países por los menos en los últimos diez años han logrado este crecimiento y ver si es algo alcanzable o... es un disparate.

\section{México crece a tasa inferior al promedio mundial}

Ilustró entonces que de los 189 países del mundo, una gran cantidad ha crecido arriba del cinco por ciento en estos diez años, cerca de la tercera parte, algunos inclusive crecen por arriba del diez por ciento. En cambio, México está creciendo a una tasa $2.6 \%$, inferior a la del promedio mundial que es de $3.8 \%$. Dicha tasa mexicana es la tasa inercial de crecimiento, 
es decir sin reformas estructurales. Dentro de esa comparación con otros países, mostró cómo México se ubica en el lugar 134 en términos de dinamismo, pero si no nos comparamos con el resto del mundo, sino solo con los países de la OCDE, estamos a media tabla, por encima de los países de los países desarrollados, pero en un lugar inferior respecto de los países emergentes. En competitividad, México ha mejorado en los últimos años pero está muy por debajo de los niveles de 2007. En productividad estamos estancados. Los precios de nuestros energéticos están evolucionando por encima de la tasa de inflación, tal es el caso del gas doméstico, la gasolina, la electricidad también está muy por encima, lo mismo que el diésel.

\section{En México, precios más caros que en Estados Unidos y peores servicios}

Eso muestra el dinamismo de los precios, pero en comparación con Estados Unidos manejamos el doble en el precio de las tarifas eléctricas, y no solo en precio sino también en calidad, es decir que en telecomunicaciones también nos comparamos de manera desfavorable respecto a Estados Unidos en cuanto que estamos muy por debajo en velocidad (megabytes por segundo: México 12.9, Estados Unidos: 24.7), en suscriptores (por 100 habitantes: México 11.9, Estados Unidos: 29.3), en conexión de fibra óptica (\% de suscriptores: México: 4.73, Estados Unidos: 7.72,) pero es

\section{En telecomunicaciones también nos comparamos de manera} desfavorable respecto a Estados Unidos. Tanto en precio como en calidad

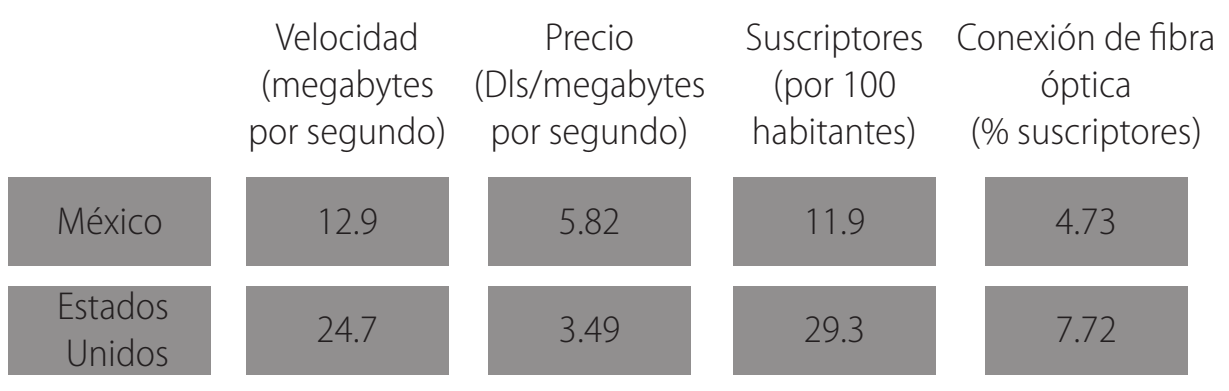

Fuente: OOKLA (2014), June, OECD Broadband Portal. 


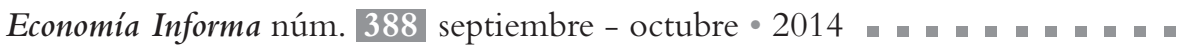

América Movil; Ganancia por suscriptor segundo trimestre de 2014 en pesos mexicanos

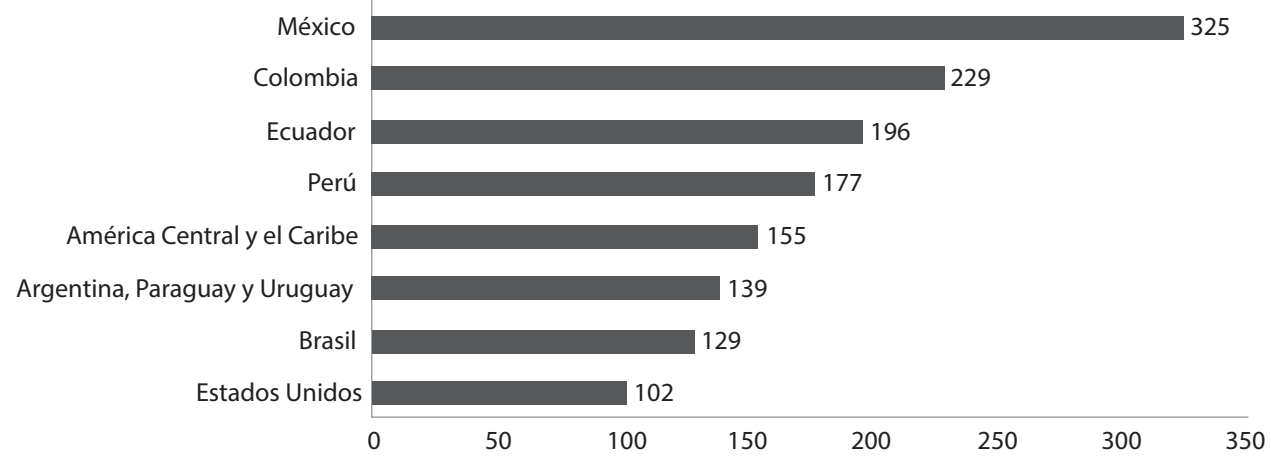

más caro el precio (dls. por megabyte por segundo: México: 5.82, Estados Unidos: 3.49).

Ejemplificó con el mayor precio de la empresa dominante, que se traduce en ganancias en servicio celular: América Móvil obtiene, en ganancias por suscriptor (al segundo trimestre de 2014 en pesos mexicanos), 325 en México, contra 102 en Estados Unidos o 129 en Brasil.

Otro dato relevante es que en recaudación tributaria con datos de 2014 de la Cepal, México ocupa el último lugar en la OECD y penúltimo en América Latina y el Caribe. En crédito interno, estamos en un lugar intermedio, pero muy lejos de otros países como Brasil.

\section{México necesitaría que Estados Unidos creciera al doble: escenario remoto}

En el escenario de las reformas estructurales y los supuestos que se deben cumplir, como el del objetivo de crecimiento de 5\%, necesitamos que la economía de Estados Unidos crezca $3.6 \%$, lo que quiere decir que necesita duplicar su crecimiento actual que es de $1.8 \%$, lo cual es bastante remoto, con todo y el buen arranque que atraviesa en estos momentos, pues su mismo gobierno plantea que promediará su crecimiento alrededor de dos por ciento. Esto es que en los últimos diez años la economía de Estados Unidos creció sólo 1.8\%, para sustentar el escenario oficial tendría que recuperar su crecimiento histórico 1947-2000 de $3.6 \%$; la CBO estima que la economía repuntará arriba de 3\% anual en un plazo corto de 2015-2017, pero posteriormente se aproximará a 2.2 por ciento.

Resumió, por lo tanto, que la viabilidad de alcanzar el crecimiento de $5 \%$ oficial es remota y depende de supuestos bastante optimistas. Apuntó que el grupo DARSI estima que con las reformas estructurales, México podrá 
pasar de un crecimiento de $2.6 \%$ a $3.4 \%$, es decir con menos optimismo que el gobierno; la tasa de desempleo se podría abatir, en números oficiales, hasta $2.7 \%$, niveles muy cercanos a los del año 2000, aunque para DARSI estarían en $3.8 \%$.

De esta forma, concluyó, la viabilidad del optimista escenario oficial a partir de las reformas, dependerá del cumplimiento simultáneo de varias condiciones: alto crecimiento de Estados Unidos, alto gasto público en infraestructura, alta inversión extranjera y de capitales, funcionamiento óptimo de reformas estructurales y ejercicio total de recursos del plan nacional de infraestructura 2014-2018.

\section{México: mercados laborales}

Finalmente y como ha sido la estrategia expositiva del Cempe (http:// www.economia.unam.mx/cempe/), Eduardo Loría desglosó su ponencia sobre la prospectiva de la economía mexicana y el tema de "México: mercados laborales" en: 1. Panorama de Estados Unidos, 2. México: principales indicadores del mercado laboral (a. Desempleo, b. Remuneraciones, c. Precariedad: subocupación e informalidad) y 3. México: Pronósticos 2014-2016.

Loría abordó lo que calificó como la parte más sensible en torno a este entorno económico, esto es: las condiciones de trabajo, el desempleo, la informalidad, lo que nos puede dar un perfil social de la visión trimestral de la economía. La primera cuestión que abordó fue la discusión sobre la presión del mercado laboral en Estados Unidos porque la tasa de desempleo estaba alrededor de 7 por ciento, es decir que muy difícilmente con todos los estímulos monetarios dados podría reducirse más. Precisó que esa reducción se debe fundamentalmente a que hay gente que ha dejado de estar en el mercado de trabajo, muchas personas han salido $(6.4 \%$ de la

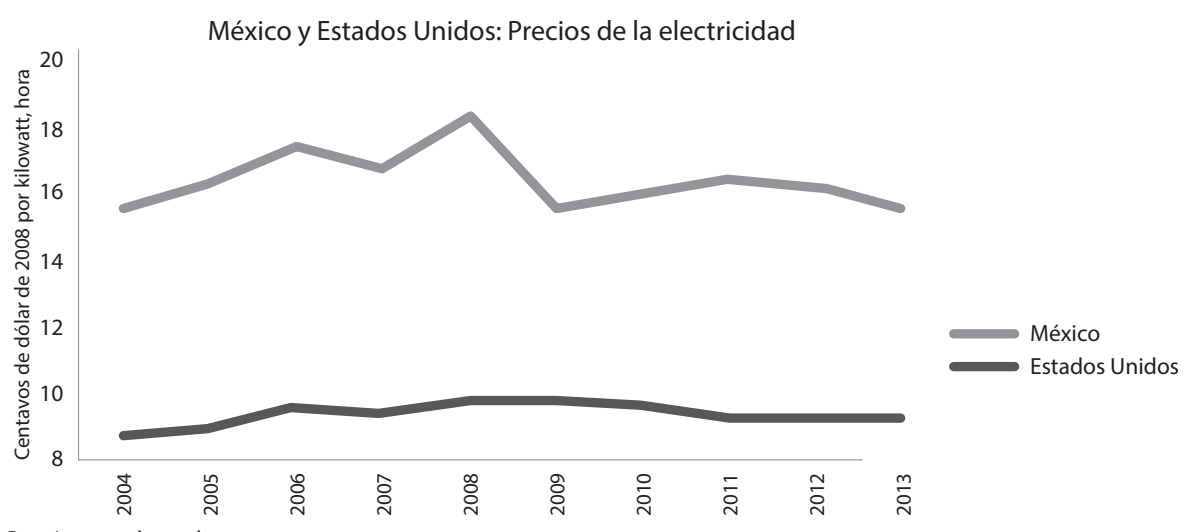

Fuente: Banxico con datos de EUA, SENER y OCDE. 
PEA) de este, y la tasa de participación es equivalente a la que había hace 37 años, y por otro lado las crisis económicas después de la segunda posguerra han sido cada vez más pronunciadas, lo cual incide en la pérdida de empleos y el tiempo en que se recuperan, como el caso de la actual crisis que implicó 84 meses para recuperar los empleos perdidos.

\section{En mujeres y jóvenes recae la precariedad laboral y el desempleo}

Por otro lado, aludió a lo que parece estar indicando un emparejamiento de la tasa de desempleo por sexo, es decir que desde la crisis de 20012003 y antes de la gran recesión de 2009, las mujeres eran mucho más desempleadas que los hombres, pero después de esta los indicadores se emparejan, aunque en las mujeres están recayendo las condiciones de precariedad laboral; una sugerencia es que las mujeres han aceptado condiciones mucho más precarias y se han ocupado en labores de menor calificación. Por grupos de edad, como se ha visto en otras presentaciones, los jóvenes son los que aumentaron dramáticamente su incidencia en el desempleo; en ellos está recayendo mayormente el problema.

\section{Condiciones críticas de ocupación}

En referencia a los salarios, después de un punto históricamente alto en 1977, no se ha recuperado, sino que aun con crecimiento económico los salarios no aumentan en proporción, pero las crisis sí han tenido efectos profundos en la caída de su valor, aunado a que el salario mínimo ha aumentado en menor medida que la inflación. Asimismo, en el tema de la precariedad del empleo, se detuvo en el concepto de "condiciones críticas de la ocupación", que se refiere a la dedicación de demasiado tiempo al trabajo (más de ocho horas), a los bajos ingresos (menos de dos salarios mínimos) o a la combinación de ambos aspectos: que trabaja demasiado y gana muy poco; esta situación, dijo, se mejoró hasta la crisis de 2009, después se detuvo y ya no ha continuado con una tendencia hacia abajo, sino que se ha mantenido.

Otro tema relevante es el de la tendencia laboral de pobreza, que tiene que ver con las personas que no pueden cubrir una canasta básica, y que se asocia con los efectos devastadores de las crisis económicas que trascienden el tiempo y llegan hasta 15 o 20 años, pues cuando por ejemplo se presenta la crisis de 2009, la 


\section{Índice de la tendencia laboral de la pobreza 2005.1-2014.1 $(2010=1)$}

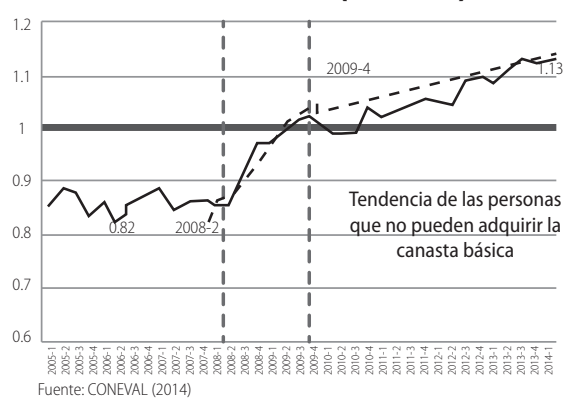

tendencia al empobrecimiento crece muy rápidamente, pero cuando la economía recupera un cierto crecimiento hasta 2012, esas condiciones de pobreza y los indicadores se han mantenido.

\section{Una buena noticia}

En otro rubro, aludió, como una buena noticia, a la recuperación de la producción industrial de Estados Unidos, a los niveles que tuvo antes de la crisis. Y los pronósticos indican que seguirán hacia arriba. Es buena noticia porque es sabido que nuestra economía está centralmente vinculada al sector industrial estadounidense. Si esto es así, tendrá un efecto positivo sobre nuestro crecimiento, no tanto como lo que se espera oficialmente, pero el crecimiento en México este año podría rondar $2.5 \%$, el año próximo cerca de $3 \%$ y el que sigue un poco arriba de tres. No ve- mos un despegue fundamental, no creemos tampoco que las reformas estructurales vayan a tener un efecto de crecimiento que saque a la economía del bache en el que estamos, pero es probable que estaremos creciendo entre 3 y 3.5 de aquí a dos años, aunque curiosamente la tasa de desempleo se mantiene estable, parecería que ha llegado a un punto que no se mueve de 5 o 5.1 .

\begin{tabular}{|l|l|l|l|}
\multicolumn{4}{|c|}{ Pronóstico básico 2014-2016 } \\
\hline PIB & 2014 & 2015 & 2016 \\
\hline I & $2.53-2.87$ & $3.19-3.47$ & 3.62 \\
\hline II & $1.89-2.54$ & $2.83-3.12$ & 3.10 \\
\hline III & $2.31-2.46$ & $3.46-3.92$ & 2.20 \\
\hline IV & $2.08-2.97$ & $3.00-3.71$ & 3.60 \\
\hline Desempleo & 2014 & 2015 & 2016 \\
\hline I & $5.1 * *$ & $5.0 * *$ & $5.1 * *$ \\
\hline II & 5.1 & 5.1 & 5.2 \\
\hline III & 5.0 & 5.0 & 5.1 \\
\hline IV & 5.2 & 5.1 & 5.1 \\
\hline
\end{tabular}

**Promedio anual.

Estimaciones realizadas es 12 de amrzo de 2014 con la colaboración de Jorge Ramírez Se autoriza da reproducción total o parcial siempre y cuando se mencione la fuente. 